\title{
Effective Wage Incentives and Binding Power of Morale
}

\author{
Ichiro Takahashi and Isamu Okada \\ Soka University, Japan
}

\begin{abstract}
The workers under consideration are motivated by free riding behavior as well as social norm. In order to seek for an optimal wage incentive system, we have constructed an agent-based model with a small group of heterogeneous or homogeneous workers. We have compared the two personnel rating systems: one based upon the level of performance relative to other workers' contemporaneous ones and the other upon her own past performance. Our simulation shows that, in inducing higher performance of the workers, the latter system excels the former for the heterogeneous organization, whereas the former system will outperform for the homogeneous one.
\end{abstract}

Keywords: agent-based simulation, wage incentive, social norm, free rider

\section{Introduction}

Kandori (2003) shows that work morale gradually erodes when there are positive externality in workers' efforts, psychological tendency with which workers abide by the social norm, and random production shocks. The novelty of the paper is that the binding power of the norm is inversely proportionate to the variance of players' effort. By constructing an artificial organization of workers with heterogeneous ability and psychological attributes, Takahashi and Okada (2004) examine the situations in which the norms and morale are subject to gradual erosion, and explore how to prevent the erosion. Virtual experiment demonstrates that financial penalty on poorly performing employees is effective to maintain the morale. They found that heterogeneous organizations are more vulnerable to morale erosion than homogeneous ones. This implies that segmentalizing an organization into small homogeneous groups will enable high performance by enhancing cohesive effect of morale. The simulation of heteroge- neous organizations reveals an interesting dynamics of workers' performances when wage incentive exists: the trend of performances oscillates.

What type of wage incentives induces high performance? To address this question, we shall compare two personnel rating systems: one is based on a worker's relative performance in a group, while the other compares an individual performance with her past performance. We will simulate the effectiveness of these rating systems. In particular we will show how the degree of heterogenuity of an organization affects the decision on the rating systems?

\section{The Model}

There are $N$ workers in a socioeconomic organization. When Worker $i$ exerts effort $e_{i} \in$ $\{0,1,2, \ldots, L\}$, she achieves ex post performance $x_{i}$ according to $x_{i}=e_{i}+u_{i}$, where $u_{i}$ is a random component (white noise) drawn out of a discrete version of uniform distribution over $\{ \pm M, \pm(M-$ $1), \ldots, 0\} . M$ is called the size of uncertainty. Let $\boldsymbol{e}=\left(e_{1}, \ldots, e_{N}\right)$ and $\boldsymbol{x}=\left(x_{1}, \ldots, x_{N}\right)$ denote profiles of effort and performance, respectively. $c(\cdot)$ is the cost function of effort, which is specified as $c\left(e_{i}\right)=\alpha e_{i}+\frac{1}{L} e_{i}^{2}$, with $\alpha$ being positive. Let $m(t)=\operatorname{median}\left\{x_{1}(t-1), \ldots, x_{N}(t-1)\right\}$ be a norm among workers, whose binding power $k(t)$ is

$$
k(t)=K\left(\sum_{i=1}^{N}\left[m(t)-x_{i}(t)\right]_{+}\right),
$$

where $[x]_{+}$denotes $\max \{0, x\} . \quad K(\cdot)$ is a nonnegative decreasing function and specified as $K(z)=\left(\frac{z}{\sigma}-1\right)^{\rho}$, where $\sigma=\frac{1}{2} L(N-1)$.

Then, $k(t)\left[m(t)-x_{i}(t)\right]_{+}$gives the psychological cost Worker $i$ incurs when her effort level falls short of the norm.

Thus, Worker $i$ 's payoff in period $t$ excluding incentive payment is given by

$$
u_{i}\left(e_{i}(t), \boldsymbol{x}(t), m(t)\right)
$$




$$
=\sum_{j=1}^{N} x_{j}(t)-c\left(e_{i}(t)\right)-k(t)\left[m(t)-x_{i}(t)\right]_{+} .
$$

We consider two types of personnel rating systems whose assessment determines the amount of reward or penalty: Group and Individual Rating Systems.

Under the Group Rating System (GRS), top $\varepsilon_{t} \%$ performers get extra $W$ dollars whereas the bottom $\varepsilon_{b} \%$ workers must pay penalty of $W$ dollars. Let $Y_{i}^{G}$ denote this (possibly negative) prize that Worker $i$ receives. Formerly, order workers' performances according to their sizes such that $x_{(1)} \leq x_{(2)} \leq \cdots \leq$ $x_{(N)}$. Then, for a given $\operatorname{GRS}\left(W, \varepsilon_{t}, \varepsilon_{b}\right)$,

$$
\begin{aligned}
& Y_{i}^{G}\left(\boldsymbol{x}(t) \mid W, \varepsilon_{t}, \varepsilon_{b}\right) \\
& =\left\{\begin{array}{cl}
W & \text { if } x_{i} \geq x_{\left(\left[N\left(1-\frac{\varepsilon_{t}}{10}\right)+1\right]\right)} \\
-W & \text { if } x_{i} \leq x_{\left(\left[N \frac{\varepsilon_{b}}{100}\right]\right)} \\
0 & \text { otherwise. }
\end{array}\right.
\end{aligned}
$$

where [ ] denotes Gauss' symbol.

Under the Individual Rating System (IRS), an individual worker is compared with her past performance. She gets extra $W$ dollars if her performance improves by more than or equal to one unit from the last period while she must pay the same amount as penalty if it worsens by $D$ units or more.

With IRS $(W, D)$,

$$
\begin{aligned}
& Y_{i}^{I}\left(x_{i}(t), x_{i}(t-1) \mid D\right) \\
& =\left\{\begin{array}{cl}
W & \text { if } x_{i}(t) \geq x_{i}(t-1)+1 \\
-W & \text { if } x_{i}(t) \leq x_{i}(t-1)-D \\
0 & \text { otherwise. }
\end{array}\right.
\end{aligned}
$$

We assume that each worker expects the other workers to select the same levels of effort as their observed performances. Moreover, the workers contemplate only the present payoff to each choice, and not the expected stream of future payoffs. These assumptions together with $E\left[x_{i}(t)\right]=e_{i}(t)$ simplifies the maximization problem of Worker $i$ 's expected payoff:

$$
\begin{aligned}
& \max _{e_{i}(t)}\left(\sum_{j \neq i} x_{j}(t-1)+e_{i}(t)-c\left(e_{i}(t)\right)\right. \\
& \left.-k(t)\left[m(t)-e_{i}(t)\right]_{+}+Y(t)\right),
\end{aligned}
$$

where $Y(t)$ is either $E\left[Y_{i}^{G} \mid e_{i}(t)\right]$ or $E\left[Y_{i}^{I} \mid e_{i}(t)\right]$.

\section{Simulation}

The parameter values are set as follows:
Periods of simulation $=100$

Maximum level of effort $(L)=100$

Initial value of effort $=0.8 \mathrm{~L}$

Size of uncertainty $(M)=2$

The power of $K(\cdot)(\rho)=32$

For each combination of experimental parameter values, we generate 30 simulations with different random seeds. There are two types of organizations. A homogeneous organization consists of 27 workers with the same productive abilitiy, i.e., $\alpha=\{1 / 2\}$, whereas a heterogeneous organization of 27 workers of low, medium, and high productive abilities: $\alpha=\{2 / 3,1 / 2,1 / 3\}$ with nine workers each. Data we shall observe in evaluating the performance of the organization would be the average effort levels of workers of each type.

\section{Results and Discussion}

First, we will observe and discuss the heterogeneous case. For each of the two rating systems, GRS and IRS, we attempt to design the best policy. Then, we will examine workers' performance for the homogeneous organization when the same best policies are applied.

\section{Heterogeneous organization}

To examine either carrot or stick is more effective in inducing higher level of performance, consider GRS with $W=0.5$. Figure 1 shows the trend of average performance of each category of workers with different incentive schemes. The parameter values $\left(\varepsilon_{t}, \varepsilon_{b}\right)$ are $(0,0),(10,10),(10,0)$ and $(0,10)$ in Figure 11 through 1-4 respectively. As seen in Figure 1-2, the oscillation occurs when both reward and punishment incentives are installed, with significantly higher performance on average than the case with neither incentive (Figure 1-1). Without the penalty incentive (Figure 1-3), the binding power is weak because low ability workers have less incentive to move upward. The trend of performance shows similar oscillating dynamics but the level itself is significantly reduced. As shown in Figure 1-4, without reward payment the trend does not oscillate because there is no strong upward movement driven by the top performers.

Thus, it appears to be important for an organization to reward the best workers to give upward momentum. To make full use of the binding power 
it is also essential to punish the least efficient workers. To sum up, in order to raise the general level of performance, the incentives for both high and low performing workers need to be installed, as in the case of Figure 1-2.

A natural question then arises: what would be the optimal level of incentives? To answer this question, we vary the amount of $W$ while keeping $\left(\varepsilon_{t}, \varepsilon_{b}\right)=(10,10)$. Figure 2 shows that as the size of $W$ increases the oscillation becomes intensified with larger amplitude although the average performance keeps rising. If we interpret the cost of effort as the magnitude of stress a worker undergos, the sharp declines in effort level may imply depression or burnout syndrome. Another problem of a large size of incentive in GRS is that it can make the workplace excessively competitive so that it will damage cooperative culture of the organization. Can we avoid these problems by adopting IRS?

First, note that each of these schemes gives quite strong incentive for workers of all types to raise their productivity. Figure $3-x$ depicts the average performance of each category of workers when $(W, D)=(0.5,2 x-1)$. When $D=1$ and $D \geq 5$, the performance trends oscillate. The mechanism working in $D=1$ case is similar as in GRS with both reward and penalty. When $\mathrm{D}=5$ or larger, workers are not afraid of being punished so that the same mechanism works as in no penalty scheme of GRS, inducing the low level of performance.

With $D=3$, there is virtually no oscillation and the average performance during the simulation period is as high as 48.34, which is even higher than 44.22 under GRS (Figure 1-2). All the type of workers are not exposed to the risk of punishment caused by adversary random shocks. This implies that there is no excessive upward momentum, which suppresses the oscillation. But reducing effort intentionally can trigger punishment when subject to negative random shock. $D=3$ (Figure $3-2$ ) is the adequate allowance in reference to the size of uncertainty $M=2$.

\section{Homogeneous organization}

We have found that Figure 1-2 and 3-2 show the trends of workers' performance under the best GRS and IRS, respectively. Figure $4-1$ and $4-2$ are the corresponding trends with the same two policies ap- plied to the homogeneous organization. The saturated levels of average performance are the same under the two systems, however, the performance of GRS consistently stays above that of IRS until they converge. This demonstrates the superiority of GRS for the organization with homogeneous workers, contrasting the superiority of IRS for the heterogeneous organization. Which of the two personnel rating system is desirable given that a manager of a firm can choose whether its organization is homogeneous or heterogeneous? Comparing Figure 3-2 and 4-1, GRS for the homogeneous organization induces higher performances than IRS for the heterogeneous one.

\section{Conclusion}

Productive effort of a worker has positive externality so that the workers under consideration are temped to be free riders. By conducting virtual simulations of an agent-based model with heterogeneous and homogeneous workers, we have examined various wage incentive systems. They show that the interaction of the binding power and workers' incentive to get reward or evade punishment generates interesting dynamics of their efforts. The two personnel rating systems are compared: one based upon the level of performance relative to other workers' performances and the other upon her own past performance. Our simulation shows that, in inducing higher performance of the workers, the latter system excels the former for the heterogeneous organization, whereas the former system will outperform for the homogeneous one.

For future extension, myopic expectation and behavior should be replaced with more sophisticated workers capable of learning.

\section{References}

[1] Kandori, M: "The Erosion and Sustainability of Norms and Morale", The Japanese Economic Review, 54, 29-48, 2003.

[2] Takahashi, I. and Isamu Okada: "Wage Incentives to Prevent the Erosion of Morale", Proceedings of the WEHIA 2004 (9th Workshop on Economics and Heterogeneous Interacting Agents), 2004. 


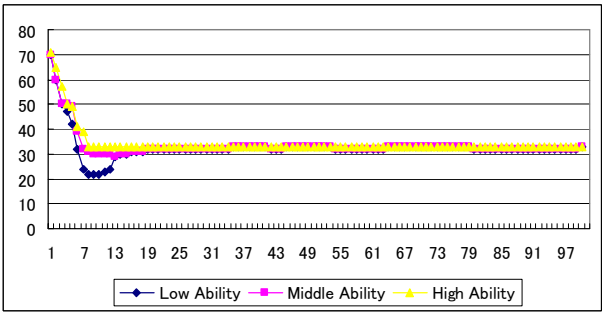

Figure 1-1

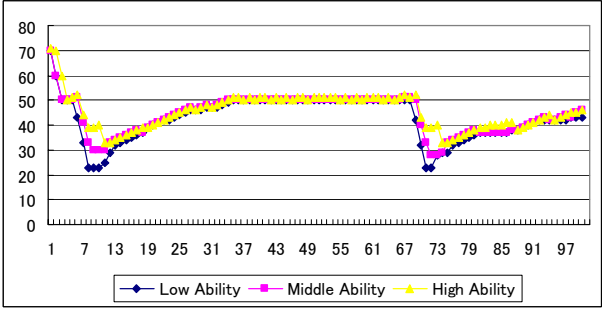

Figure 1-2

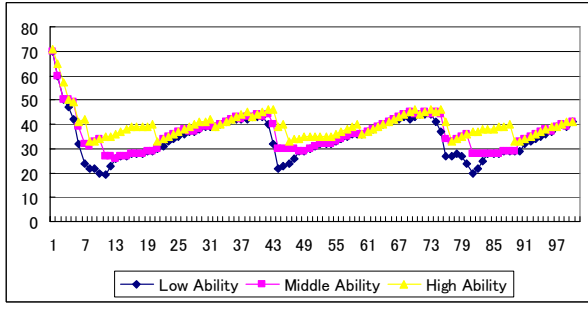

Figure 1-3

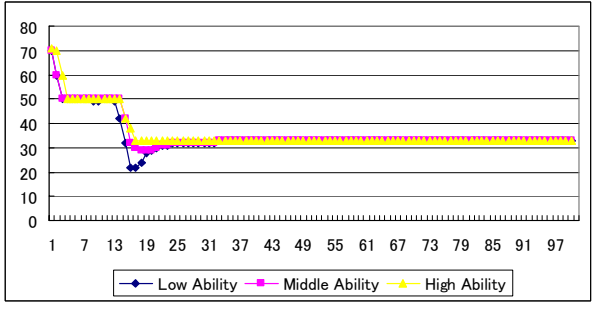

Figure 1-4

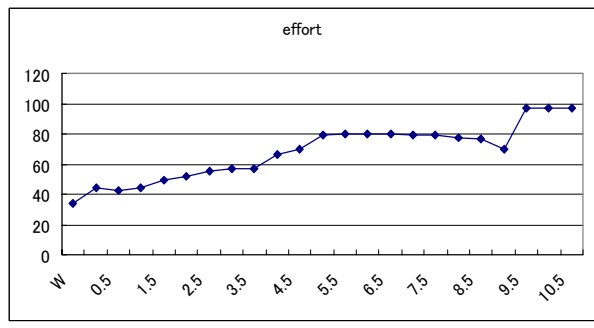

Figure 2

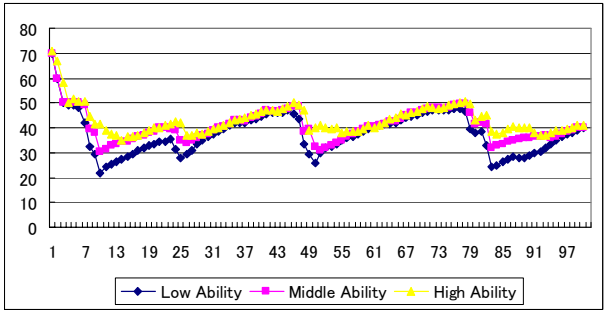

Figure 3-1

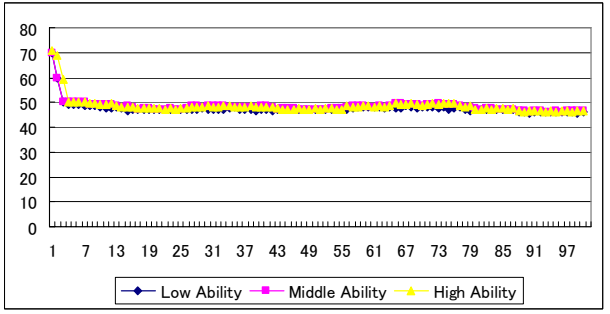

Figure 3-2

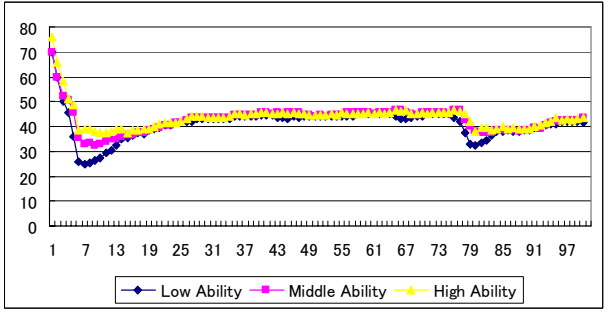

Figure 3-3

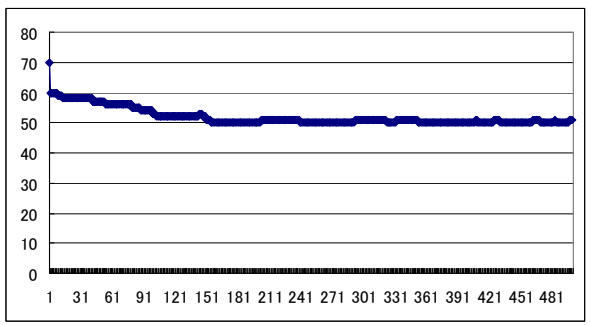

Figure 4-1

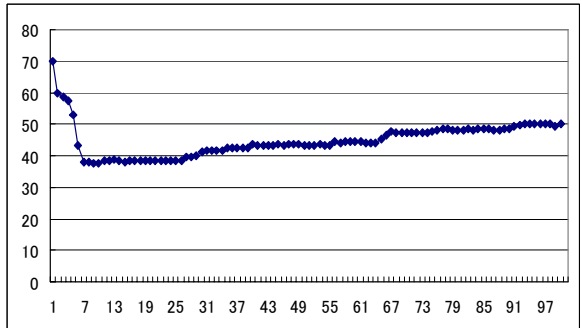

Figure 4-2 